\title{
CXC195 induces apoptosis and endoplastic reticulum stress in human hepatocellular carcinoma cells by inhibiting the PI3K/Akt/mTOR signaling pathway
}

\author{
XIAO-LIANG CHEN ${ }^{1,2^{*}}, \mathrm{JIAN}^{\mathrm{P} I N G \mathrm{FU}^{2 *}, \mathrm{JUN} \mathrm{SHI}^{3}, \mathrm{PING}^{2} \mathrm{WAN}^{2}, \mathrm{HONG} \mathrm{CAO}^{2} \text { and ZHI-MOU TANG }}{ }^{4}$ \\ ${ }^{1}$ Department of Surgery, School of Medicine, Nanchang University; ${ }^{2}$ Department of Hepatobiliary Surgery, \\ Jiangxi Provincial People's Hospital; ${ }^{3}$ Department of Hepatobiliary Surgery, The First Affiliated Hospital of Nanchang University; \\ ${ }^{4}$ Department of Oncology, Jiangxi Provincial People's Hospital, Nanchang, Jiangxi 330006, P.R. China
}

Received December 18, 2014; Accepted September 16, 2015

DOI: $10.3892 / \mathrm{mmr} .2015 .4479$

\begin{abstract}
CXC195 exhibits strong protective effects against neuronal apoptosis by exerting antioxidant activity. However, the pharmacological function of CXC195 in cancer remains to be elucidated. The present study demonstrated that CXC195 exhibited significant cytotoxic effects, and induced cell cycle arrest and apoptosis in HepG2 human hepatocellular carcinoma (HCC) cell lines. Following treatment of HepG2 cells with $150 \mu \mathrm{M}$ CXC195 for 24 , cell viability and the apoptotic rate were assessed using an MTT assay and Annexin V/propidium iodide staining followed by flow cytometric analysis. Molecular markers of the cell cycle, apoptosis, mitochondrial function and endoplasmic reticulum (ER) stress were analyzed by western blot or polymerase chain reaction analysis. Caspase activation, cytochrome $c$ and apoptosis-inducing factor release, and analysis of the B cell lymphoma 2 (Bcl-2)-associated X protein/Bcl-2 ratio demonstrated that the anticancer effects of CXC195 in HepG2 cells were mediated by caspase and mitochondria-dependent apoptosis. CXC195 also induced the expression of ER stress-associated proteins, including CCAAT-enhancer-binding protein homologous protein, and glucose-regulated proteins 94 and 78, and led to the activation of multiple branches of ER stress transducers, including inositol-requiring enzyme $1 \alpha$-apoptosis signal-regulating kinase-p38/c-Jun N-terminal kinase, and protein kinase R-like endoplasmic reticulum kinase-eukaryotic translation initiation factor $2 \alpha$-activating transcription factor (ATF) 4 and ATF6,
\end{abstract}

Correspondence to: Professor Zhi-Mou Tang, Department of Oncology, Jiangxi Provincial People's Hospital, 92 Aiguo Road, Nanchang, Jiangxi 330006, P.R. China

E-mail: tangzhimouoncology@163.com

*Contributed equally

Key words: CXC195, hepatocellular carcinoma, apoptosis, endoplasmic reticulum stress, phosphoinositide 3-kinase/protein kinase $\mathrm{B} /$ mammalian target of rapamycin in the HepG2 cells. In addition, CXC195 inhibited the phosphorylation of phosphoinositide 3-kinase (PI3K), Akt and mammalian target of rapamycin (mTOR) in the HepG2 cells. These effects were enhanced following treatment with selected inhibitors of PI3K (LY294002), Akt (SH-6) and mTOR (rapamycin). Furthermore, these inhibitors enhanced the pro-apoptotic effects of CXC195 in the HepG2 cells. In conclusion, the results of the present study indicated that CXC195 induced apoptosis and ER stress in HepG2 cells through the inhibition of the PI3K/Akt/mTOR signaling pathway.

\section{Introduction}

Hepatocellular carcinoma (HCC), a primary hepatic tumor with aggressive malignancy and high prevalence, is the third leading cause of cancer-associated mortality worldwide $(1,2)$. Although progress in HCC treatment has been achieved during the last three decades, patients with advanced and terminal stages of HCC continue to have a poor prognosis, owing to the paucity of effective and tolerable systemic chemotherapy strategies $(3,4)$. Due to the multi-fold molecular pathogenesis and high chemoresistance of HCC, as well as the low selectivity of chemotherapeutic drugs, it is essential to develop multi-target compounds, which can target several aberrant signaling pathways concurrently, which may not only improve medication efficacy, but also minimize drug toxicity $(5,6)$.

Multiple pathophysiological processes, including viral replication, protein overloading, oxidative stress and the inflammatory reaction, are involved in the oncogenesis of HCC caused by chronic liver diseases, including hepatitis B and $\mathrm{C}$ viral infection, alcoholic cirrhosis and obesity-associated non-alcoholic fatty liver disease (7). Notably, these events in hepatocytes induce a state of stress in the endoplasmic reticulum (ER), termed ER stress $(8,9)$. There has been increasing awareness with regards to the role of ER stress in the homeostasis of cancer cells $(5,9)$. ER stress occurs when ER homeostasis is lost due to an overload of protein folding in the ER. Abnormalities in ER function can cause ER stress, which results in an unfolded protein response (UPR), including three key signaling proteins: Inositol-requiring enzyme $1 \alpha$ (IRE1 $\alpha$ )-apoptosis signal-regulating kinase (ASK)-p38/c-Jun 
$\mathrm{N}$-terminal kinase (JNK), protein kinase R-like endoplasmic reticulum kinase (PERK)-eukaryotic translation initiation factor $2 \alpha$ (eIF2 $\alpha$ )-activating transcription factor (ATF) 4 and ATF6, which act as the sensors of ER stresses. Furthermore, several mechanisms have been suggested to link ER stress with apoptosis, including the B cell lymphoma 2 (Bcl-2) and caspase family $(10,11)$.

Tetramethylpyrazine (TMP) is one of the major bioactive components purified from the Chinese herb, Chuanxiong (Ligusticum wallichi Franchat), and has been widely used in the treatment of various types of human cancer, including lung cancer (12), osteosarcoma (13), gliomas $(14,15)$, ovarian carcinoma (16), breast cancer (17), gastric cancer (18) and HCC (19). CXC195 is a TMP analogue, which can protect human umbilical vein endothelial cells from $\mathrm{H}_{2} \mathrm{O}_{2}$-induced apoptosis through inhibition of the mitochondria- and caspase 3 -dependent signaling pathways $(20,21)$. In addition, CXC195 exhibits antioxidant activity and anti-apoptotic effects in transient focal ischemia by inhibiting the expression levels of NADPH oxidase and nitric oxide synthase (22), and by regulating the PI3K/Akt/glycogen synthase kinase $3 \beta$ signaling pathway (23). Despite evidence indicating the anticancer effects of TMP, there is a lack of data describing the anticancer activity of its analogue, CXC195. The present study examined whether CXC195 induced apoptosis and ER stress in human HCC HepG2 cells, and investigated the possible mechanisms underlying these effects.

\section{Materials and methods}

Cell lines and culture. Human HCC HepG2 cells were purchased from the American Type Culture Collection (Manassas, VA, USA) and maintained in Dulbecco's modified Eagle's medium (Invitrogen; Thermo Fisher Scientific., Inc., Waltham, MA, USA) supplemented with $10 \%$ heat-inactivated fetal bovine serum (Invitrogen; Thermo Fisher Scientific., Inc.), $100 \mathrm{U} / \mathrm{ml}$ penicillin (Invitrogen) and $10 \mu \mathrm{g} / \mathrm{ml}$ streptomycin (Invitrogen) at $37^{\circ} \mathrm{C}$ in an atmosphere containing $5 \% \mathrm{CO}_{2}$. The media were replaced every 2-3 days and sub-cultured when the cell population density reached 70-80\% confluence. CXC195 (Fig. 1A) was synthesized by the direct reaction of 2-chloromethyl-3,5,6-trimethylpyrazine hydrochloride (Sigma-Aldrich, St. Louis, MO, USA) with 4,4'-difluorobenzhydrylpiperazine (SigmaAldrich). The purity of CXC195 (>98\%) was determined using high-performance liquid chromatography (Waters, Milford, MA, USA). The examined compounds and positive control were dissolved in $0.1 \%$ dimethylsulfoxide (DMSO; SigmaAldrich)). LY294002, SH-6 and rapamycin were purchased from Santa Cruz Biotechnology, Inc. (Dallas, TX, USA).

Cell viability assay. Cell viability was assessed using an MTT assay (Santa Cruz Biotechnology, Inc.). The spent medium was removed and $10 \mu \mathrm{l}$ MTT solution $(5 \mathrm{mg} / \mathrm{ml})$ was added to $100 \mu \mathrm{l}$ respective growth medium without phenol red, and the plates containing $1 \times 10^{6}$ cells/well were incubated at $37^{\circ} \mathrm{C}$ for $4 \mathrm{~h}$ in a humidified atmosphere of $5 \% \mathrm{CO}_{2}$. The formazan crystals formed by the mitochondrial reduction of MTT were solubilized in DMSO (100 $\mu \mathrm{l} /$ well $)$ and the absorbance was read at $540 \mathrm{~nm}$ using a microplate reader (Bio-Rad Laboratories, Inc., Hercules, CA, USA).
Double staining for Annexin V-fluorescein isothiocyanate (FITC) and propidium iodide (PI). Briefly, the HepG2 cells were detached with $0.25 \%$ trypsin (Invitrogen) and washed twice with phosphate-buffered saline (PBS), followed by centrifugation at $12,000 \times \mathrm{g}$ for $5 \mathrm{~min}$. A total of $1 \times 10^{6}$ cells were then suspended in $1.25 \mathrm{mM}$ extracellular calcium $(131 \mathrm{mM} \mathrm{NaCl}$, $5 \mathrm{mM} \mathrm{KCl}, 1.3 \mathrm{mM} \mathrm{MgSO}_{4}, 1.3 \mathrm{mM} \mathrm{CaCl}_{2}, 0.4 \mathrm{mM} \mathrm{KH}_{2} \mathrm{PO}_{4}$, $6 \mathrm{mM}$ glucose, $20 \mathrm{mM}$ HEPES; pH 7.4) and double-stained with Annexin V-FITC and PI (1:100; BD Biosciences, San Jose, CA, USA) at $4^{\circ} \mathrm{C}$ for $30 \mathrm{~min}$ at room temperature. The fluorescence of each sample was subsequently quantitatively analyzed using a FACSCalibur flow cytometer (BD Biosciences) and CellQuest software (version 5.1; BD Biosciences).

Measurement of the cell cycle. The cells were treated with $20 \mu \mathrm{g} / \mathrm{ml}$ RNase A (Invitrogen), followed by $25 \mu \mathrm{g} / \mathrm{ml}$ PI. The cell population at each stage of the cell cycle was then determined by examining the intensity of PI fluorescence on a flow cytometer using an argon laser and a $570 \mathrm{~nm}$ bandpass filter (FACSort; BD Biosciences).

Caspase 3, 8, and 9 activity assays. HepG2 cells were gently lysed in ice-cold lysis buffer [250 mM sucrose, $1 \mathrm{mM}$ EDTA, $0.05 \%$ digitonin, $25 \mathrm{mM}$ Tris ( $\mathrm{pH}$ 6.8), $1 \mathrm{mM}$ dithiothreitol, $1 \mu \mathrm{g} / \mathrm{ml}$ leupeptin, $1 \mu \mathrm{g} / \mathrm{ml}$ pepstatin, $1 \mu \mathrm{g} / \mathrm{ml}$ aprotinin, $1 \mathrm{mM}$ benzamidine and $0.1 \mathrm{mM}$ phenylmethylsulphonyl fluoride] for $30 \mathrm{~min}$, and centrifuged at $12,000 \mathrm{xg}$ at $4^{\circ} \mathrm{C}$. The cell lysates $(30 \mu \mathrm{g})$ from the HepG2 cells were determined spectrophotometrically at $405 \mathrm{~nm}$ using a microtiter plate reader (model M100L; Microfluidics, Newton, MA, USA). The assays to determine the activities of caspase 3,8 and 9 were performed by incubating the cell lysates with $0.2 \mathrm{mM}$ of one of the following caspase-specific colorimetric tetrapeptide substrates: Ac-DEVD-pNA for caspase 3, Ac-IETD-pNA for caspase 8 and Ac-LEHD-p-nitroaniline (pNA) for caspase 9, for $1 \mathrm{~h}$ at $37^{\circ} \mathrm{C}$, as previously described (24). The increase in the absorbance at $405 \mathrm{~nm}$, which corresponds to the quantity of pNA released from the peptide substrates was then converted into units of enzyme activity using a standard curve generated with free pNA. One unit of caspase 3,8, or 9 activity corresponded to the quantity of enzyme that releases $1 \mathrm{pmol} / \mathrm{min}$ pNA from 0.2 mM DEVD-pNA, IETD-pNA or Ac-LEHD-pNA, respectively. Lysates from HepG2 cells treated with DMSO were also used in these assays, which served as a control group.

Determining the release of cytochrome $c(C y t-c)$ and apoptosis-inducing factor (AIF). The HepG2 cells were collected by centrifugation at $12,000 \mathrm{xg}$ at $4^{\circ} \mathrm{C}$ for $5 \mathrm{~min}$ at $48^{\circ} \mathrm{C}$, and washed with ice-cold PBS. Subsequent fractionation of the mitochondrial and cytosolic proteins were performed using a Mitochondrial Protein Extraction kit (Santa Cruz Biotechnology, Inc.), according to the manufacturer's protocol. The cell nuclear and cytosolic fractions were prepared using a Nuclear/Cytosol Fractionation kit, purchased from BioVision Inc. (Milpitas, CA, USA), according to the manufacturer's protocol. Following centrifugation, the supernatants were obtained for western blot analysis.

Western blot analysis. HepG2 cells were gently lysed in ice-cold lysis buffer [250 mM sucrose, $1 \mathrm{mM}$ EDTA, $0.05 \%$ 
A
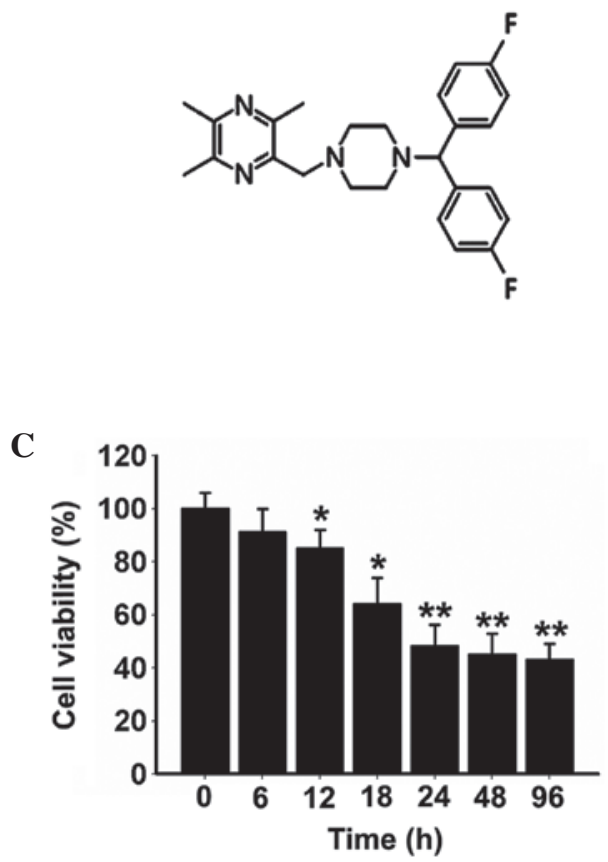

B

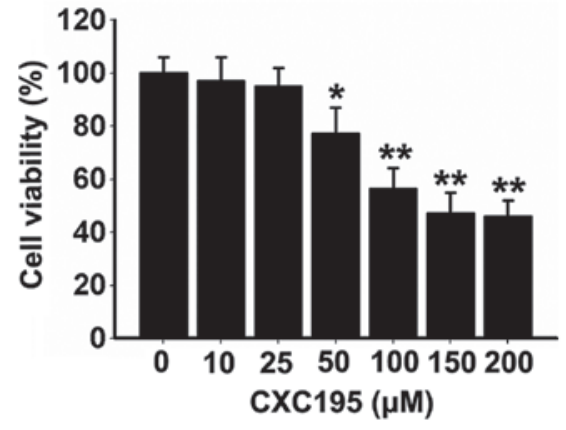

D

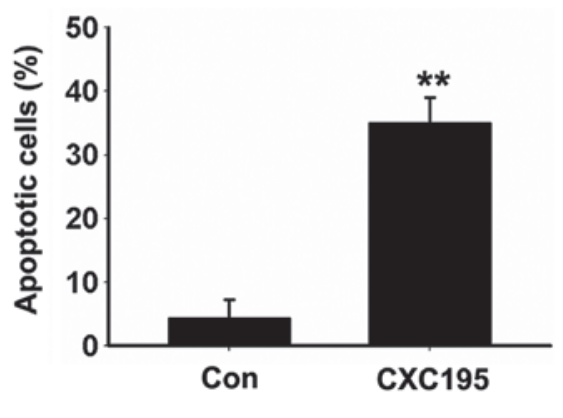

Figure 1. Effects of CXC195 on the viability and apoptotic rates of HepG2 cells. (A) Chemical structure of CXC195. Cell viability was determined using an MTT assay. (B) Effects of various doses of CXC195 on the cell viability of HepG2 cells. The control group was set at 100\%. (C) Time-dependent effects of treatment with $150 \mu \mathrm{M}$ CXC195 on the viability of HepG2 cells. HepG2 cells treated with $150 \mu \mathrm{M} \mathrm{CXC195}$ for $0 \mathrm{~h}$ was set at $100 \%$. (D) Effects of $150 \mu \mathrm{M}$ CXC195 on the apoptotic rates of HepG2 cells. The cells were analyzed using flow cytometry following staining with Annexin V and propidium iodide. Data are presented as the mean \pm standard deviation $(n=6)$. ${ }^{*} \mathrm{P}<0.05$ and ${ }^{* *} \mathrm{P}<0.01$, vs. control. Con, control.

digitonin, $25 \mathrm{mM}$ Tris (pH 6.8), $1 \mathrm{mM}$ dithiothreitol, $1 \mu \mathrm{g} / \mathrm{ml}$ leupeptin, $1 \mu \mathrm{g} / \mathrm{ml}$ pepstatin, $1 \mu \mathrm{g} / \mathrm{ml}$ aprotinin, $1 \mathrm{mM}$ benzamidine and $0.1 \mathrm{mM}$ phenylmethylsulphonyl fluoride] for $30 \mathrm{~min}$ and centrifuged at $12,000 \mathrm{xg}$ at $4^{\circ} \mathrm{C}$. Protein samples from the HepG2 cells extracts were separated using $8 \%$ SDS-PAGE (high molecular weight) or 10\% SDS-PAGE (regular molecular weight) (Sigma-Aldrich) and transferred onto a nitrocellulose membrane (GE Healthcare Life Sciences, Little Chalfont, UK). The membrane was blocked with 5\% skimmed milk and incubated with PBS containing Tween 20 and the following primary antibodies at 1:500 dilution: Monoclonal/polyclonal mouse/rabbit anti-human proliferating cell nuclear antigen (PCNA) (cat no. sc-25280), p21 (sc-397), p27 (sc-393380), p53 (sc-126), Bcl-2 (sc-7382), Bcl-2-associated X (Bax) (sc-7480), AIF (sc-13116), Cyt-c (sc-13561), cyclooxygenase (COX)4 (sc-292052), tubulin (sc-9104), histone (sc-10806), glucose-regulated protein (GRP)94 (sc-11402), GRP78 (sc-1050), CCAAT-enhancer-binding protein homologous protein (CHOP) (sc-575) and GAPDH (sc-365062) (Santa Cruz Biotechnology, Dallas, TX, USA) and cyclin-dependend kinase (CDK)4 (cat no. 12790), cyclin D1 (1044), phosphorylated (p)-PERK (3179), PERK (3192), eIF2 $\alpha$ (9722), p-eIF2 $\alpha$ (3597), ATF4 (11815), IRE1 $\alpha$ (3294), p-ASK (3765), ASK (37626S), p-p38 (4511), p38 (9213), ATF6 (11815S), p-PI3K (4288S), PI3K (4249S), p-AKT (4060P), AKT (\#2920S), mammalian target of rapamycin (mTOR) (2983S) and p-mTOR (5536S) (Cell Signaling Technology, Inc., Danvers, MA, USA) overnight at $4^{\circ} \mathrm{C}$. After washing with Tris-buffered saline containing 1\% Tween 20 (Sigma-Aldrich), membranes were incubated with the horseradish peroxidase-conjugated: Goat anti-rabbit immunoglobulin (Ig)G (cat. no. sc-34661) or donkey anti-goat
IgG (cat. no. sc-362265; Santa Cruz Biotechnology, Inc.) for $2 \mathrm{~h}$ at $4^{\circ} \mathrm{C}$. The blots were developed using enhanced chemiluminescence western blotting detection reagents (Santa Cruz Biotechnology, Inc.). Densitometric analysis of the bands was performed using Image Master ${ }^{\mathrm{TM}}$ 2D Elite 3.1 software (GE Healthcare Life Sciences).

Statistical analysis. Values are expressed as the mean \pm standard deviation. Differences were analyzed using an unpaired Student's t-test and one-way analysis of variance. All data were analyzed using Graph Prism Software version 5 (GraphPad Inc., La Jolla, CA, USA). $\mathrm{P}<0.05$ was considered to indicate a statistically significant difference.

\section{Results}

Effects of CXC195 on the proliferation of HepG2 cells. The present study first investigated the antiproliferative effects of CXC195 on HepG2 cells at various concentrations (10, 25, 50, $100,150$ and $200 \mu \mathrm{M})$ and time points $(6,12,18,24,48$ and 96 h) using an MTT assay. As shown in Fig. 1B, HepG2 cell viability decreased gradually following treatment with $50 \mu \mathrm{M}$ CXC195 for $24 \mathrm{~h}$, and was maintained at its lowest levels when treated with 150-200 $\mu \mathrm{M}$ CXC195 for $24 \mathrm{~h}$. Treatment with $150 \mu \mathrm{M}$ CXC195 induced a marked downregulation in cell viability, in a time-dependent manner, with maximum inhibition of cell viability detected between 24 and 96 h (Fig. 1C). Therefore, treatment with $150 \mu \mathrm{M} \mathrm{CXC195}$ for $24 \mathrm{~h}$ was selected for the subsequent experiments.

Annexin V/PI staining was used to evaluate the effects of CXC195 on the type of cell death. As shown in Fig. 1D, 
A

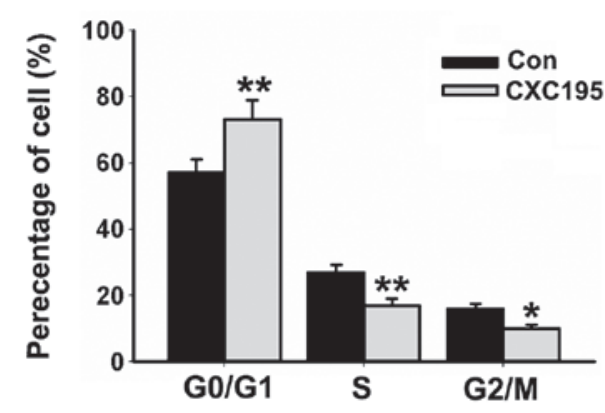

C
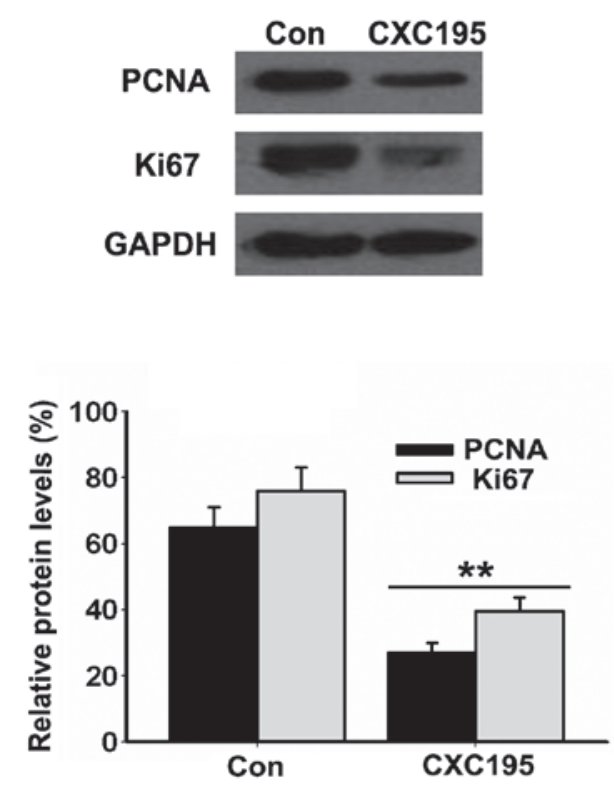

B
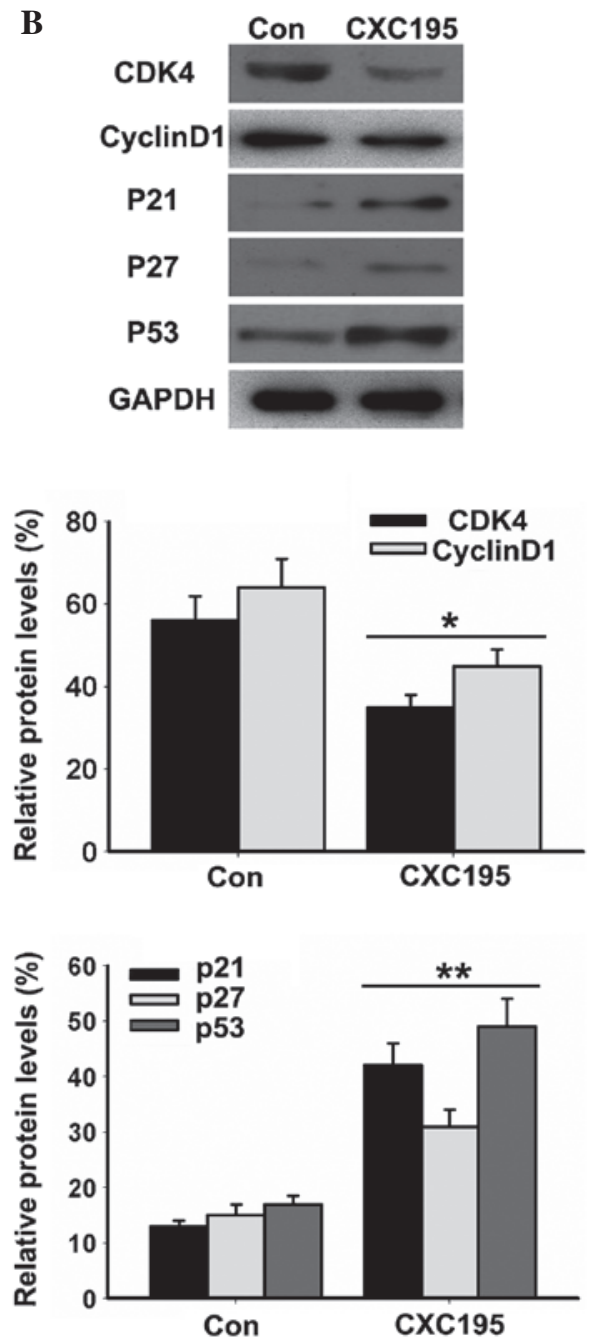

Figure 2. Effects of CXC195 on the proliferation of HepG2 cells. (A) Cell cycle distribution was examined using flow cytometry. (B and C) Protein expression levels of PCNA, Ki67, CDK4, cyclin D1, p21, p27 and p53 were determined using western blot analysis. GAPDH was used to confirm equal protein loading. Data are presented as the mean \pm standard deviation $(n=6)$. ${ }^{*} \mathrm{P}<0.05$ and ${ }^{* *} \mathrm{P}<0.01$, vs. control. PCNA, proliferating cell nuclear antigen; $\mathrm{CDK} 4$, cyclin-dependent kinase 4; Con, control.
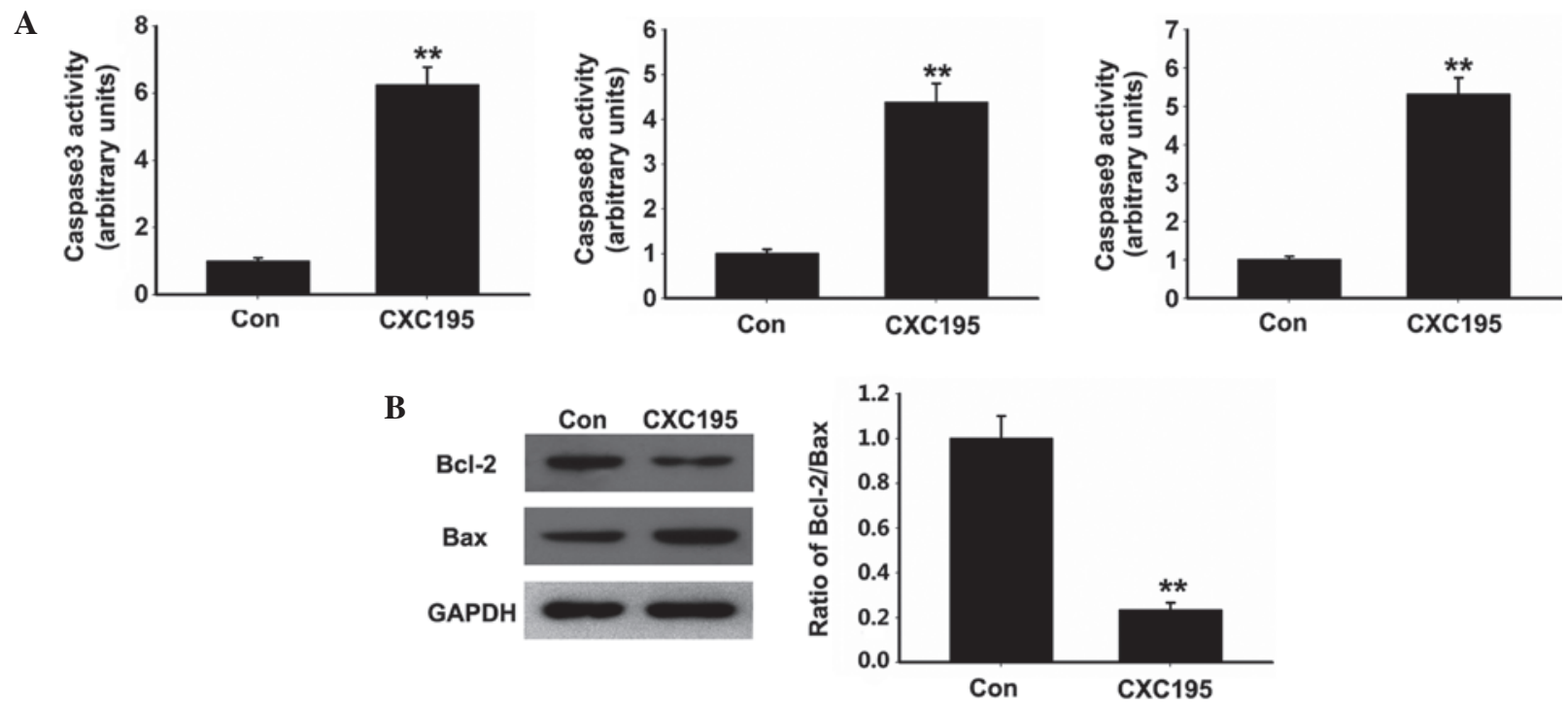

Figure 3. Effects of CXC195 on the caspase- and mitochondria-dependent apoptotic signaling pathways in HepG2 cells. The HepG2 cells were treated with $150 \mu \mathrm{M} \mathrm{CXC195}$ for $24 \mathrm{~h}$. (A) Activities of caspase 3, 8 and 9 were measured in the HepG2 cells and the (B) protein expression levels of Bcl-2 and Bax were detected using western blot analysis, and the ratio of Bax/Bcl-2 was determined following treatment of the HepG2 cells with CXC195. GAPDH was used to confirm equal protein loading. Data are presented as the mean \pm standard error of the mean $(n=6)$. ${ }^{* *} \mathrm{P}<0.01$, vs. control. Bcl-2, B cell lymphoma 2; Bax, Bcl-2-associated X protein; Con, control. 


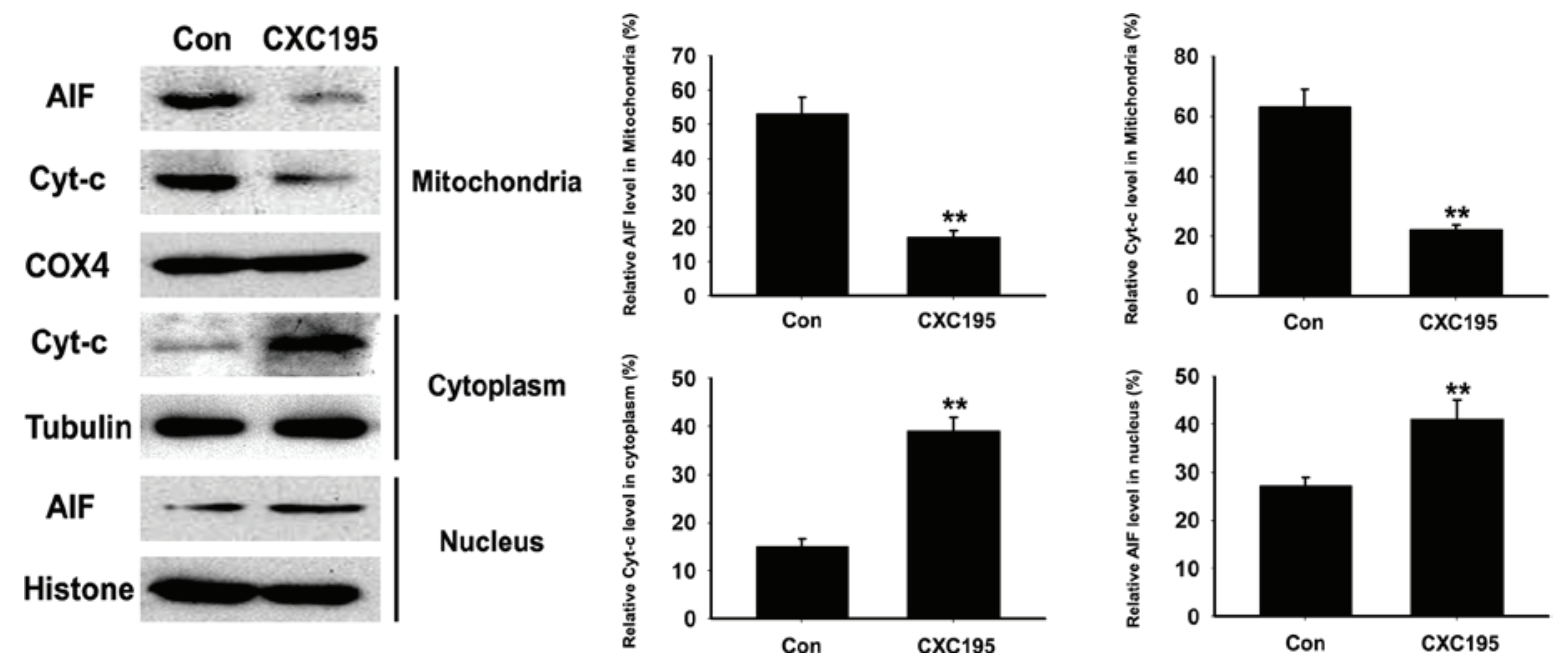

Figure 4. Effects of CXC195 on the release of Cyt- $c$ in HepG2 cells. The expression levels of Cyt- $c$ in mitochondrial and cytosolic fractions, and the expression levels of AIF in the mitochondrial and nuclear fractions were measured in the HepG2 cells using western blotting. The expression levels of COX4, tubulin and histone (an internal control) were used to monitor Cyt $c$ and AIF release. Data are presented as the mean \pm standard error of the mean $(n=6){ }^{* *} \mathrm{P}<0.01$, vs. control. Cyt- $c$, cytochrome $c$; AIF, apoptosis-inducing factor; $\mathrm{COX} 4$, cytochrome $c$ oxidase subunit 4 .
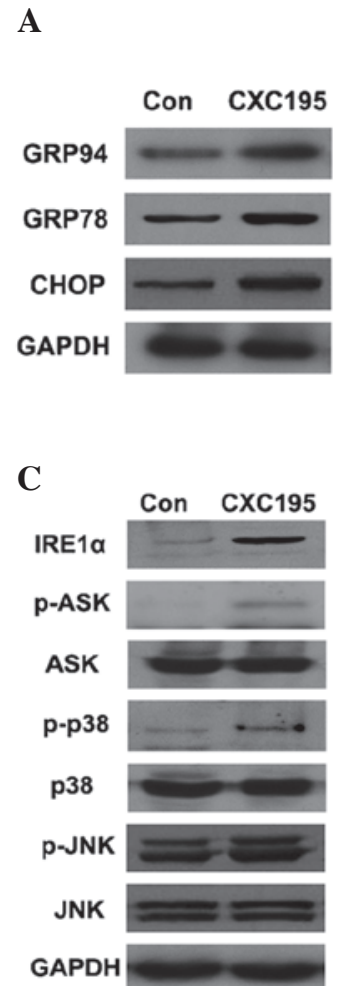
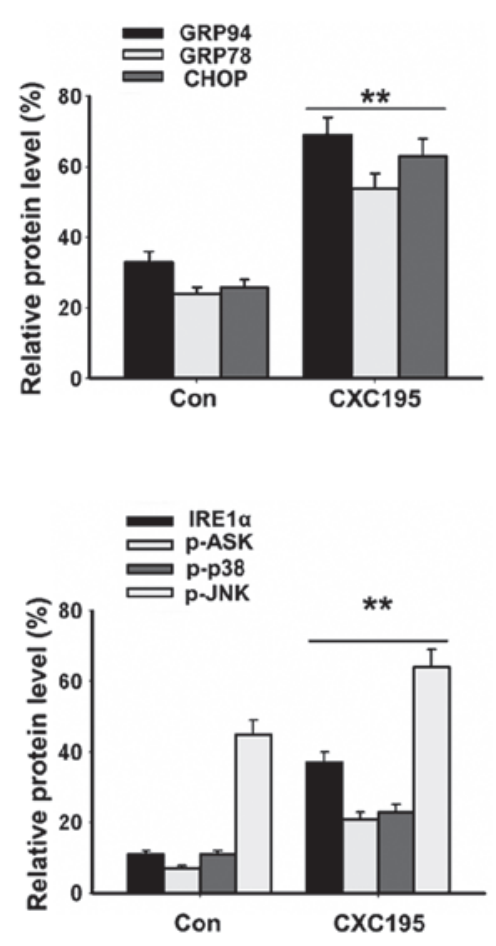

B

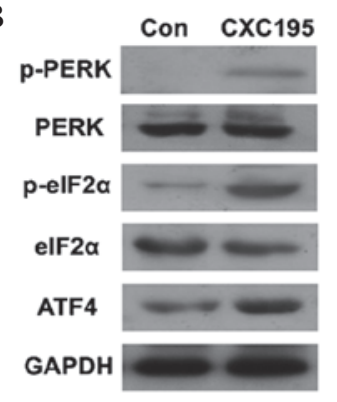

D
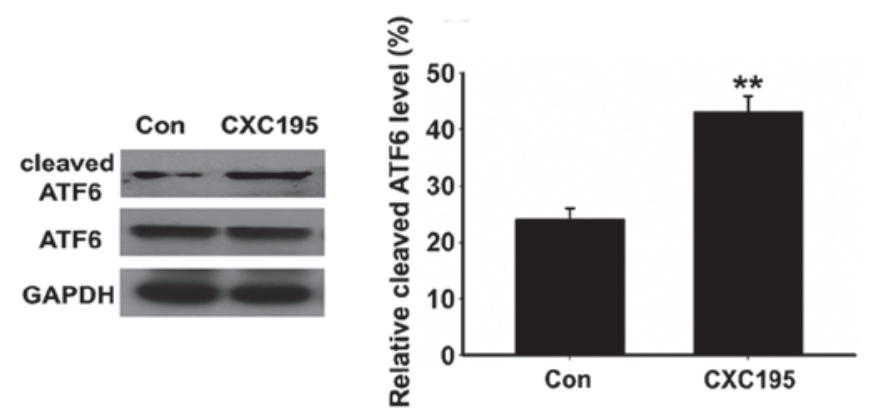

Figure 5. Effects of CXC195 on endoplasmic reticulum stress in the HepG2 cells. The HepG2 cells were treated with $150 \mu \mathrm{M}$ CXC195 for 24 h. (A) Expression levels of GRP78, GRP94 and CHOP were measured in the HepG2 cells using western blot analysis. (B) Expression levels of p-PERK, PERK, p-eIF2 $\alpha$, eIF2 $\alpha$ and ATF4 were measured in the HepG2 cells using western blot analysis. (C) Expression levels of IRE1 $\alpha$, p-ASK, ASK, p-p38, p38, p-JNK and JNK were measured in the HepG2 cells using western blot analysis. (D) Expression of cleaved ATF6 was measured in the HepG2 cells using western blot analysis. GAPDH was used to confirm equal protein loading. Data are presented as the mean \pm standard deviation $(n=6) .{ }^{* *} \mathrm{P}<0.01$, vs. control. GRP, glucose-regulated protein; CHOP, CCAAT-enhancer-binding protein homologous protein; p-, phosphorylated; PERK, protein kinase R-like endoplasmic reticulum kinase; eIF2 $\alpha$, eukaryotic translation initiation factor $2 \alpha$; AIF4, apoptosis-inducing factor 4; IRE1 $\alpha$, inositol-requiring enzyme 1 $\alpha$; ASK, apoptosis signal-regulating kinase; JNK, c-Jun N-terminal kinase; ATF6, activating transcription factor 6; Con, control.

CXC195 significantly increased the rate of apoptosis in the HepG2 cells. Cell cycle analysis demonstrated that CXC195 inhibited cell cycle progression of the HepG2 cells, by increasing the proportion of cells in the $G_{0} / G_{1}$ phase and decreasing the proportion of cells in the $S$ and $G_{2} / M$ phases
(Fig. 2A). The results also demonstrated that CXC195 significantly inhibited the expression levels of the CDK4 and cyclin D1 cell cycle-associated proteins (Fig. 2B), and the PCNA and Ki67 markers of cell proliferation (Fig. 2C). By contrast, treatment with CXC195 elevated the expression levels of the 
A
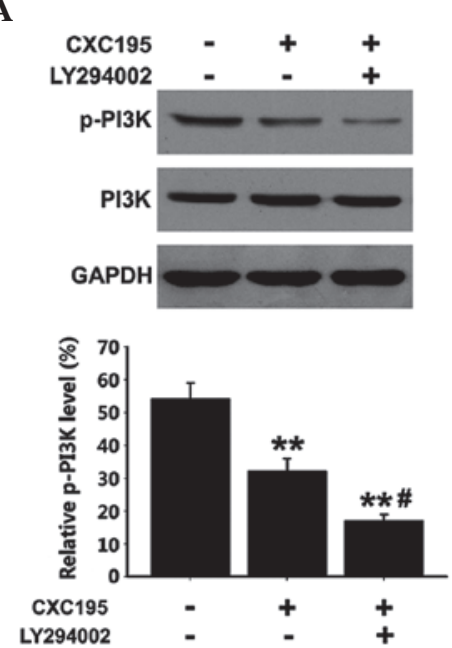

D

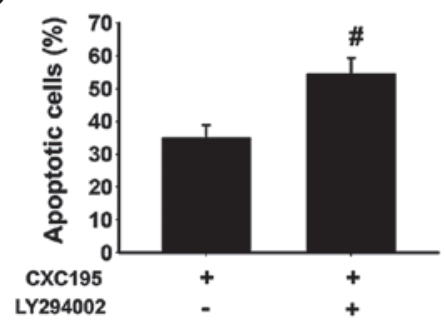

B
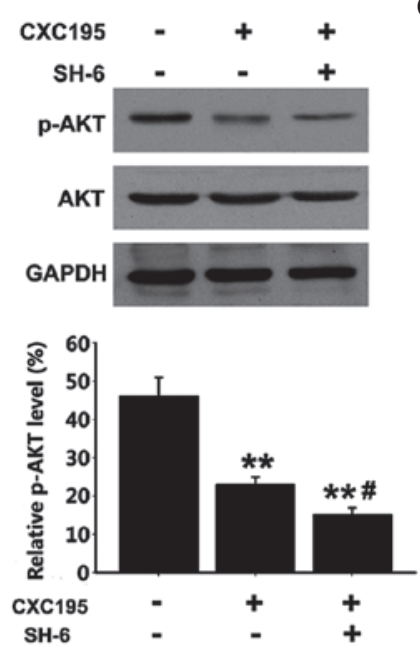

E

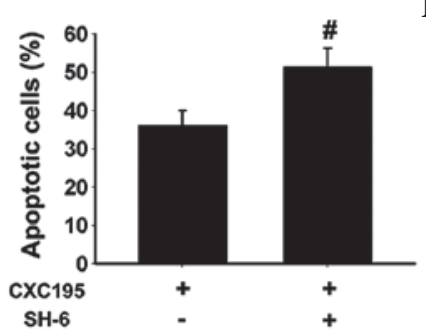

C
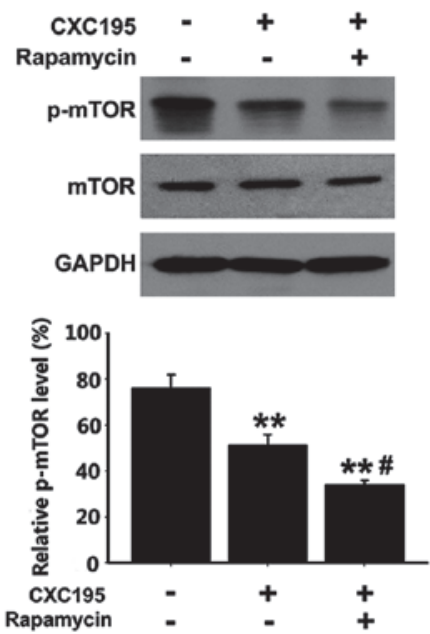

F

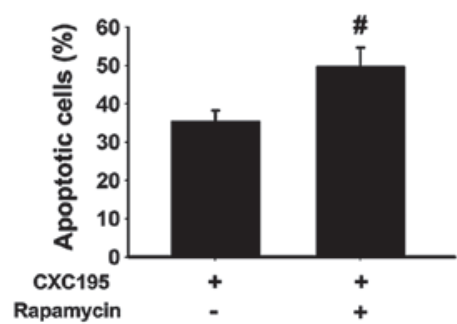

Figure 6. Effects of CXC195 on the PI3K/Akt/mTOR signaling pathway in HepG2 cells. (A) Expression levels of p-PI3K were measured in the HepG2 cells following treatment with $150 \mu \mathrm{M}$ CXC195 and the selective PI3K inhibitor (LY294002) for $24 \mathrm{~h}$ using western blot analysis. (B) Expression levels of p-Akt were measured in the HepG2 cells following treatment with $150 \mu \mathrm{M} \mathrm{CXC195}$ and the selective Akt inhibitor (SH-6) using western blot analysis. (C) Expression levels of p-mTOR were measured in the HepG2 cells following treatment with $150 \mu \mathrm{M} \mathrm{CXC195}$ and the selective mTOR inhibitor (rapamycin) for $24 \mathrm{~h}$ using western blot analysis. The bar charts show the ratio of p-PI3K, p-Akt and p-mTOR to GAPDH in the HepG2 cells. (D) Effects of LY294002 on the levels of apoptosis in HepG2 cells following treatment with $150 \mu \mathrm{M} \mathrm{CXC195.} \mathrm{(E)} \mathrm{Effects} \mathrm{of} \mathrm{SH-6} \mathrm{on} \mathrm{the} \mathrm{levels} \mathrm{of} \mathrm{apoptosis} \mathrm{in} \mathrm{HepG2} \mathrm{cells} \mathrm{following} \mathrm{treatment} \mathrm{with}$ $150 \mu \mathrm{M}$ CXC195. (F) Effects of rapamycin on the levels of apoptosis in HepG2 cells following treatment with $150 \mu \mathrm{M}$ CXC195. HepG2 cells treated with LY294002/SH-6/rapamycin and CXC195 exhibited a significant increase in the apoptotic ratio compared with that of cells treated with CXC195 only. Data are presented as the mean \pm standard error of the mean $(\mathrm{n}=6) .{ }^{* *} \mathrm{P}<0.01$, vs. untreated control; ${ }^{*} \mathrm{P}<0.01$, vs. cells treated with $\mathrm{CXC} 195$ without inhibitor. PI3K, phosphoinositide 3-kinase; Akt, protein kinase B; mTOR, mammalian target of rapamycin; p, phosphorylated.

p21, p27 and p53 CDK inhibitors (Fig. 2C) in the HepG2 cells. These results suggested that $\mathrm{CXC195}$ inhibited the proliferation and induced the apoptosis of the HepG2 cells.

Effects of CXC195 on the caspase and mitochondria-dependent signaling pathway in HepG2 cells. Apoptosis induced by various cytotoxic agents is dependent on the activation of caspases, which are important in cleaving specific target proteins (25). Therefore, the present study assessed whether CXC195 activated caspase signaling pathways in the HepG2 cells. As shown in Fig. 3A, CXC195 was found to increase the activities of caspase 3,8 and 9 following treatment of the HepG 2 cells with $150 \mu \mathrm{M}$ CXC195 for $24 \mathrm{~h}$.

As Bcl-2 family proteins are reported to regulate the mitochondria-mediated apoptosis signaling pathway by maintaining a balance between pro- and anti-apoptotic members (26), the present study examined the effects of CXC195 on the expression levels of Bcl-2 family proteins in the HepG2 cells. CXC195 increased the protein expression of pro-apoptotic Bax, but decreased the protein expression of $\mathrm{Bcl}-2$ in the HepG 2 cells. In addition, the $\mathrm{Bcl}-2 / \mathrm{Bax}$ ratio was decreased in the HepG2 cells following treatment with CXC195 (Fig. 3B).
The release of pro-apoptotic proteins, including Cyt- $c$ and AIF, from the intermembrane space into the cytosol is required for caspase activation, which initiates the apoptotic program (27). As shown in Fig. 4, following treatment with CXC195, the expression levels of Cyt- $c$ and AIF in the HepG2 cells significantly decreased in the mitochondria, whereas the expression of Cyt- $c$ increased significantly in the cytosol. AIF can translocate from the cytosol to the nucleus, where it is involved in chromatinolysis (27); therefore, the present study investigated whether CXC195 induces the relocation of AIF to the nucleus. As shown in Fig. 4, the expression of AIF was detected in the nuclei of the CXC195-treated HepG2 cells, whereas minimal AIF was detected in the untreated HepG2 cells. These data suggested that CXC195 induced HepG2 cell apoptosis through caspase and mitochondria-dependent mechanisms.

Effects of CXC195 on ER stress in HepG2 cells. There is increasing evidence that ER stress is crucial in the regulation of apoptosis $(8,9)$. To confirm the hypothesis that ER stress is involved in mediating the apoptotic effects of CXC195, the effect of CXC195 on the expression levels of the ER stress-associated proteins, GRP78, GRP94 and CHOP, were 
examined in the HepG2 cells. Western blotting demonstrated that the expression levels of GRP78, GRP94 and CHOP increased following treatment with $150 \mu \mathrm{M} \mathrm{CXC195}$ for $24 \mathrm{~h}$ (Fig. 5A). ER stress induced three key signaling pathways: IRE1 $\alpha$-ASK-P38/JNK, PERK-eIF2 $\alpha$-ATF4 and ATF6. As shown in Fig. 5B-D, CXC195 increased the expression levels of p-PERK, p-eIF2 $\alpha$, p-ASK, p-p38 and p-JNK, and induced the activation of ATF4, IRE1 $\alpha$ and ATF6 in the HepG2 cells. These results suggested that ER stress is involved in the CXC195-induced apoptosis of HepG2 cells.

Effects ofCXC195 onPI3K/Akt/mTORactivationinHepG2 cells. To further understand the molecular mechanisms underlying the induction of apoptosis in HepG2 cells following treatment with CXC195, the expression of PI3K, Akt and mTOR, which are critical signaling proteins associated with cell apoptosis, were examined. As shown in Fig. 6A-C, the protein expression levels of p-PI3K, p-Akt and p-mTOR were determined using western blot analysis, which demonstrated that CXC195 significantly inhibited the phosphorylation of PI3K at Tyr458, Akt at Ser473 and mTOR at Ser2448. The HepG2 cells were also treated with either the selective PI3K inhibitor, LY294002, the selective Akt inhibitor, SH-6, or the selective mTOR inhibitor, rapamycin. The selective inhibitors were observed to further inhibit the phosphorylation of PI3K, Akt and mTOR in the CXC195-treated HepG2 cells. Furthermore, the HepG2 cells treated with LY294002/SH-6/rapamycin and CXC195 exhibited significant increases in apoptosis, compared with the cells treated with CXC195 only (Fig. 6D-F). These results indicated that the pro-apoptotic effects of CXC195 in HepG2 cells were associated with inhibition of the PI3K/Akt/mTOR signaling cascade.

\section{Discussion}

Apoptosis is defined as programmed cell death and has been suggested as an efficient antitumor mechanism (25). Malignant tumor cells can be eliminated following treatment with anticancer chemotherapies though apoptosis (28), and apoptosis can be controlled by extrinsic and intrinsic signaling pathways (29). The extrinsic signaling pathway involves the death receptor, in which the death domains target caspase 8 . The activation of caspase 8 subsequently activates caspase 3 , which induces apoptosis (29). Caspase 3 is a member of the caspase family of enzymes, which are major inducers of apoptosis, and the levels of caspase 3 activity are often measured in investigations into antitumor drugs targeting apoptosis (29). In the present study, the activation of caspases 3 and 8 were induced following treatment with $150 \mu \mathrm{M}$ CXC195 for $24 \mathrm{~h}$. The results indicated that CXC195 induced HepG2 cell apoptosis through the regulation of the extrinsic signaling pathway.

Members of the Bcl-2 family (intrinsic or mitochondrial signaling pathway) are also key regulators of apoptosis and the intrinsic pathway of apoptosis is associated with DNA damage. Oligomerization of Bax or Bak, which are pro-apoptotic proteins of the Bcl-2 family, promotes the release of mitochondrial Cyt- $c$ into the cytoplasm, which combines with the caspase 9 precursor to form an apoptosis complex, and the activation of caspase 9 activates caspase 3 to induce apoptosis (30). The results of the present study demonstrated that decreased expression of anti-apoptotic Bcl-2 and increased expression of pro-apoptotic Bax were observed in the CXC195-treated HepG2 cells. In addition, the activation of caspase 9 was induced following treatment with CXC195. Furthermore, the levels of Cyt- $c$ and AIF significantly decreased in the mitochondria, whereas the level of Cyt- $c$ increased in the cytosol, and that of AIF increased in the nucleus. Therefore, CXC195 may have initiated the intrinsic signaling pathway, which induced caspase-dependent apoptotic signaling in the HepG2 cells.

The Bcl-2 family proteins also localise to the ER during stress, where their suggested functions include the regulation of apoptosis and the UPR $(10,11)$. ER stress occurs when ER homeostasis is lost due to an overload of protein folding in the ER, and the UPR induces the upregulation of ER-resident chaperones, including GRP78 and GRP94 $(8,9)$. The results of the present study demonstrated that CXC195 increased the expression levels of GRP78 and GRP94 in the HepG2 cells. Abnormalities in ER function can cause ER stress, which results in the UPR and includes three key signaling pathways: IRE1 $\alpha$-ASK-P38/JNK, PERK-eIF2 $\alpha$-ATF4 and ATF6, which act as the sensors of ER stresses $(8,9)$. In the present study, CXC195 increased the activation of these signaling pathways in the HepG2 cells. The GRP78 ER stress sensor and three branches of the UPR pathway, together with the CHOP mediator constitute an essential and dominant arm, and the interaction between this arm and TRB3 has been demonstrated in wild-type HepG 2 cells. Previous studies have reported that the UPR pathway was the candidate pathway owing to its ability to initiate CHOP-mediated programmed cell death, which is independent of the activation of the intrinsic and extrinsic apoptotic signaling pathways $(31,32)$. In agreement with the effect of CXC195 on ER stress in the present study, CXC195 induced increased expression levels of CHOP in the HepG2 cells. These results indicated that ER stress contributed to the pro-apoptotic effect of CXC195 in HepG2 cells.

The PI3K-Akt-mTOR signaling pathway is one of the most important signaling pathways in the regulation of cell proliferation, growth and apoptosis in various types of cancer, as it increases the activity of anti-apoptotic Akt $(33,34)$. The phosphorylation of PI3K, Akt and mTOR is required for the suppression of cancer cell apoptosis and tumor progression (35). The PI3K/Akt/mTOR signaling pathway inhibits apoptosis by inactivating important members of the apoptotic cascade, including caspase 3 and 9, Bax and Bad $(35,36)$. UPR initiates programmed cell death through the transcriptional regulation of genes involved in cell death, including Bcl-2, Bim, DR5 and TRB3. TRB3 is a pseudokinase, which inhibits the activation of Akt by directly binding to Akt and preventing the phosphorylation at Ser473 of Akt (36). Therefore, there is cross-talk between the UPR and Akt signaling pathways. The data obtained in the present study revealed that CXC195 downregulated the phosphorylation of PI3K, Akt and mTOR, whereas the inhibitors of these proteins (LY294002, SH-6 and rapamycin, respectively) enhanced the inhibitory effects of CXC195 in the HepG2 cells. In addition, these inhibitors increased the number of apoptotic HepG2 cells induced by CXC195. Overall, these results $t$ indicated that the pro-apoptotic effects of CXC195 in HepG2 cells were associated with inhibiting the activation of the PI3K-Akt-mTOR signaling cascade. 
In conclusion, the present study reported for the first time, to the best of our knowledge, that CXC195 inhibited the proliferation and induced the apoptosis of HepG2 cells, which was mediated through the caspase-, mitochondrial- and ER stress-dependent signaling pathways. In addition, CXC195 caused inactivation of the PI3K-Akt-mTOR signaling pathway, which may exhibit a critical function in CXC195-induced apoptosis in HepG2 cells.

\section{References}

1. Jemal A, Bray F, Center MM, Ferlay J, Ward E and Forman D: Global cancer statistics. CA Cancer J Clin 61: 69-90, 2011.

2. El-Serag HB and Rudolph KL: Hepatocellular carcinoma: Epidemiology and molecular carcinogenesis. Gastroenterology 132: 2557-2576, 2007.

3. Bruix J and Llovet JM: Major achievements in hepatocellular carcinoma. Lancet 373: 614-616, 2009.

4. Whittaker S, Marais R and Zhu AX: The role of signaling pathways in the development and treatment of hepatocellular carcinoma. Oncogene 29: 4989-5005, 2010.

5. Wei L, Lu N, Dai Q, Rong J, Chen Y, Li Z, You Q and Guo Q: Different apoptotic effects of wogonin via induction of $\mathrm{H}(2)$ $\mathrm{O}(2)$ generation and $\mathrm{Ca}(2+)$ overload in malignant hepatoma and normal hepatic cells. J Cell Biochem 111: 1629-1641, 2010.

6. Llovet JM, Burroughs A and Bruix J: Hepatocellular carcinoma. Lancet 362: 1907-1917, 2003.

7. Dara L, Ji C and Kaplowitz N: The contribution of endoplasmic reticulum stress to liver diseases. Hepatology 53: 1752-1763, 2011

8. Malhi $\mathrm{H}$ and Kaufman RJ: Endoplasmic reticulum stress in liver disease. J Hepatol 54: 795-809, 2011.

9. Xu M, Lu N, Zhang H, Dai Q, Wei L, Li Z, You Q and Guo Q: Wogonin induced cytotoxicity in human hepatocellular carcinoma cells by activation of unfolded protein response and inactivation of Akt. Hepatol Res 43: 890-905, 2013.

10. Oakes SA, Lin SS and Bassik MC: The control of endoplasmic reticulum-initiated apoptosis by the BCL-2 family of proteins. Curr Mol Med 6: 99-109, 2006.

11. Rong Y and Distelhorst CW: Bcl-2 protein family members: Versatile regulators of calcium signaling in cell survival and apoptosis. Annu Rev Physiol 70: 73-91, 2008.

12. Zheng CY, Xiao W, Zhu MX, Pan XJ, Yang ZH and Zhou SY: Inhibition of cyclooxygenase-2 by tetramethylpyrazine and its effects on A549 cell invasion and metastasis. Int J Oncol 40: 2029-2037, 2012.

13. Wang Y, Fu Q and Zhao W: Tetramethylpyrazine inhibits osteosarcoma cell proliferation via downregulation of $\mathrm{NF}-\kappa \mathrm{B}$ in vitro and in vivo. Mol Med Rep 8: 984-988, 2013.

14. Yu K, Chen Z, Pan X, Yang Y, Tian S, Zhang J, Ge J, Ambati B and Zhuang J: Tetramethylpyrazine-mediated suppression of C6 gliomas involves inhibition of chemokine receptor CXCR4 expression. Oncol Rep 28: 955-960, 2012.

15. Fu YS, Lin YY, Chou SC, Tsai TH, Kao LS, Hsu SY, Cheng FC, Shih YH, Cheng H, Fu YY and Wang JY: Tetramethylpyrazine inhibits activities of glioma cells and glutamate neuro-excitotoxicity: Potential therapeutic application for treatment of gliomas. Neuro Oncol 10: 139-152, 2008.

16. Yin J, Yu C, Yang Z, He JL, Chen WJ, Liu HZ, Li WM, Liu HT and Wang YX: Tetramethylpyrazine inhibits migration of SKOV3 human ovarian carcinoma cells and decreases the expression of interleukin-8 via the ERK1/2, p38 and AP-1 signaling pathways. Oncol Rep 26: 671-679, 2011

17. Zhang Y, Liu X, Zuo T, Liu Y and Zhang JH: Tetramethylpyrazine reverses multidrug resistance in breast cancer cells through regulating the expression and function of P-glycoprotein. Med Oncol 29: 534-538, 2012.
18. Yi B, Liu D, He M, Li Q, Liu T and Shao J: Role of the ROS/AMPK signaling pathway in tetramethylpyrazine-induced apoptosis in gastric cancer cells. Oncol Lett 6: 583-589, 2013.

19. Wang XB, Wang SS, Zhang QF, Liu M, Li HL, Liu Y, Wang JN, Zheng F, Guo LY and Xiang JZ: Inhibition of tetramethylpyrazine on P-gp, MRP2, MRP3 and MRP5 in multidrug resistant human hepatocellular carcinoma cells. Oncol Rep 23: 211-215, 2010

20. Cheng XC, Liu XY, Xu WF, Guo XL and Ou Y: Design, synthesis and biological activities of novel ligustrazine derivatives. Bioorg Med Chem 15: 3315-3320, 2007.

21. Ou Y, Dong X, Liu XY, Cheng XC, Cheng YN, Yu LG and Guo XL: Mechanism of tetramethylpyrazine analogue CXC195 inhibition of hydrogen peroxide-induced apoptosis in human endothelial cells. Biol Pharm Bull 33: 432-438, 2010.

22. Liu H, Wei $X$, Chen L, Liu X, Li S, Liu $X$ and Zhang X: Tetramethylpyrazine analogue CXC195 protects against cerebral ischemia/reperfusion injury in the rat by an antioxidant action via inhibition of NADPH oxidase and iNOS expression. Pharmacology 92: 198-206, 2013.

23. Chen L, Wei X, Hou Y, Liu X, Li S, Sun B, Liu X and Liu H: Tetramethylpyrazine analogue CXC195 protects against cerebral ischemia/reperfusion-induced apoptosis through PI3K/Akt/GSK3 $\beta$ pathway in rats. Neurochem Int 66: 27-32, 2014.

24. Masoud L, Vijayasarathy C, Fernandez-Cabezudo M, Petroianu G and Saleh AM: Effect of malathion on apoptosis of murine L929 fibroblasts: A possible mechanism for toxicity in low dose exposure. Toxicology 185: 89-102, 2003.

25. Kuwana $\mathrm{T}$ and Newmeyer DD: Bcl-2-family proteins and the role of mitochondria in apoptosis. Curr Opin Cell Biol 15: 691-699, 2003.

26. Loeffler M and Kroemer G: The mitochondrion in cell death control: Certainties and incognita. Exp Cell Res 256: 19-26, 2000.

27. Palanca JM, Aguirre-Rueda D, Granell MV, Aldasoro M, Garcia A, Iradi A, Obrador E, Mauricio MD, Vila J, Gil-Bisquert A and Valles SL: Sugammadex, a neuromuscular blockade reversal agent, causes neuronal apoptosis in primary cultures. Int J Med Sci 10: 1278-1285, 2013.

28. Hannun YA: Apoptosis and the dilemma of cancer chemotherapy. Blood 89: 1845-1853, 1997.

29. Chiantore MV, Vannucchi S, Mangino G, Percario ZA, Affabris E, Fiorucci G and Romeo G: Senescence and cell death pathways and their role in cancer therapeutic outcome. Curr Med Chem 16: 287-300, 2009.

30. He K, Si P, Wang H, Tahir U, Chen K, Xiao J, Duan X, Huang R and Xiang G: Crocetin induces apoptosis of BGC-823 human gastric cancer cells. Mol Med Rep 9: 521-526, 2014.

31. Rao RV, Ellerby HM and Bredesen DE: Coupling endoplasmic reticulum stress to the cell death program. Cell Death Differ 11: 372-380, 2004.

32. Oyadomari S and Mori M: Roles of CHOP/GADD153 in endoplasmic reticulum stress. Cell Death Differ 11: 381-389, 2004.

33. Huang KF, Zhang GD, Huang YQ and Diao Y: Wogonin induces apoptosis and down-regulates survivin in human breast cancer MCF-7 cells by modulating PI3K-Akt pathway. Int Immunopharmacol 12: 334-341, 2012.

34. Sui T, Ma L, Bai X, Li Q and Xu X: Resveratrol inhibits the phosphatidylinositide 3-kinase/protein kinase $\mathrm{B} / \mathrm{mammalian}$ target of rapamycin signaling pathway in the human chronic myeloid leukemia K562 cell line. Oncol Lett 7: 2093-2098, 2014.

35. Luo J, Manning BD and Cantley LC: Targeting the PI3K-Akt pathway in human cancer: Rationale and promise. Cancer cell 4: 257-262, 2003

36. Yamaguchi $\mathrm{H}$ and Wang HG: CHOP is involved in endoplasmic reticulum stress-induced apoptosis by enhancing DR5 expression in human carcinoma cells. J Biol Chem 279: 45495-45502, 2004. 